\title{
Unexpected massive bleeding caused by extensive maxillary osteonecrosis in a breast cancer patient: a case report
}

\author{
Bailong Liu ${ }^{1 \#}$, Yunfei Ma ${ }^{1 \#}$, Hui Liu ${ }^{1 \#}$, Chong Wang ${ }^{2}$, Liang Guo ${ }^{3}$, Aiping Shi ${ }^{4}$, Min Liu ${ }^{1}$ \\ ${ }^{1}$ Department of Radiation Oncology, The First Hospital of Jilin University, Changchun, China; ${ }^{2}$ Department of Hepatobiliary and Pancreatic \\ Medicine, The First Hospital of Jilin University, Changchun, China; ${ }^{3}$ Department of Pathology, The First Hospital of Jilin University, Changchun, \\ China; ${ }^{4}$ Department of Breast Surgery, The First Hospital of Jilin University, Changchun, China \\ \#These authors contributed equally to this work. \\ Correspondence to: Aiping Shi, MD, PhD. Department of Breast Surgery, The First Hospital of Jilin University, 1 Xinmin Street, Changchun, 130021, \\ China. Email: sap@jlu.edu.cn; Min Liu, MD, PhD. Department of Radiation Oncology, The First Hospital of Jilin University, 1 Xinmin Street, \\ Changchun 130021, China. Email: lmin99@jlu.edu.cn.
}

\begin{abstract}
Diphosphonate application is routinely recommended to treat bone metastasis (BM) in cancer patients. However, the severe side effects of diphosphonate, especially after long-term use, are often overlooked by clinicians. In this article, we describe a case in which a heavily-treated breast cancer patient, suffered from massive bleeding as a result of maxillary osteonecrosis by zoledronic acid (ZA) and apatinib. In October 2018, a 48-year-old Chinese female with breast cancer presented at our department with brain metastases. The patient had experienced progression multiple times and had received several lines of systemic interventions. ZA was administered monthly for a rather long period of 37 months. She also took $250 \mathrm{mg}$ of apatinib, a small molecular tyrosine kinase inhibitor (TKI) that targets vascular endothelial growth factor receptor 2, daily for 11 days. However, massive bleeding from the oral and nasal cavity occurred that could not be alleviated by conventional means. Computed tomography revealed severe destruction and loss of the right maxillary bone and maxillary sinus wall. A pathological examination of the exfoliated bone tissue further confirmed that the patient was suffering from necrosis rather than metastasis. An emergency interventional embolization was performed, and the bleeding was stopped. In this case, maxillary osteonecrosis developed from the antiresorptive agents. Antiangiogenesis drugs should be avoided whenever possible. In clinical practice, the high risk of osteonecrosis needs to be carefully considered. Further, care needs to be taken to ensure osteonecrosis is not misdiagnosed as BM, especially in stage IV patients. Pathology is a prerequisite for the timely and correct diagnosis and management. Life-threatening toxicity such as massive bleeding, should be avoided to ensure that patients receive adequate antitumor treatments.
\end{abstract}

Keywords: Massive bleeding; osteonecrosis; zoledronic acid (ZA); antiangiogenesis; case report

Submitted Mar 05, 2021. Accepted for publication Aug 26, 2021.

doi: $10.21037 /$ tcr-21-404

View this article at: https://dx.doi.org/10.21037/tcr-21-404

\section{Introduction}

Due to their efficacy in reducing osteolysis, bisphosphonates (BPs) are attractive agents for bone metastasis (BM) in malignant tumors. Zoledronic acid (ZA), a thirdgeneration nitrogen-containing $\mathrm{BP}$ has been reported to exert pleiotropic antitumor effects in vitro (1). Thus, the long-term application of $\mathrm{BP}$ is very common in clinical settings. Antiangiogenetic drugs, including both monoclonal antibodies and small molecule tyrosine kinase inhibitors (TKIs), produce promising effects when used in monotherapy or combined with other antitumor approaches, such as chemotherapy, immune checkpoint inhibitors, and radiotherapy. In metastatic and refractory settings, the combined application of antiresorptive and antiangiogenetic agents is common. However, the severe 
complications this combination causes, have been largely ignored. Previous studies have shown that the use of BPs to prevent and treat bone-related events in patients with tumor or osteoporosis can cause medicine-related osteonecrosis of the jaw (MRONJ), which may increase when used in combination with antiangiogenic drugs (2). In the case presented here, massive bleeding resulted from osteonecrosis in a heavily treated breast cancer patient. Additionally, the mechanism by which $\mathrm{BP}$ and the antiangiogenetic drugs induced osteonecrosis was analyzed. This case study suggests that clinicians should make further efforts to ensure the early detection and appropriate management of osteonecrosis. We believe that this case raises the issue of whether severe osteonecrosis can be avoided in heavily-treated cancer populations. We present the following case in accordance with the CARE reporting checklist (available at https://dx.doi.org/10.21037/tcr-21-404).

\section{Case presentation}

In May 2014, a 44-year-old Chinese woman presented with a 3-year history of a painless mass in her left breast. Her past medical and family history was unremarkable. The mass progressed from $1.5 \times 1.5$ to $4.0 \times 4.0 \mathrm{~cm}$ in size at the physical examinations. Ultrasound demonstrated an irregular hypoechoic lesion measuring about $39.1 \mathrm{~mm} \times$ $25.9 \mathrm{~mm}$ in size located in the outer upper quadrant of the left breast. Additionally, several axillary lymph nodes were found in the left, some of which had lost normal structure, and the largest of which was about $13.8 \mathrm{~mm} \times 10.5 \mathrm{~mm}$ in size. A subsequent biopsy of the breast tumor verified invasive ductal breast carcinoma with immunohistochemical results of estrogen receptor (ER) $(80 \%+)$, progesterone receptor $(\mathrm{PR})(80 \%+)$, human epidermal growth factor receptor 2 (HER2) (3+), and Ki-67 (20\%+). A biopsy of the left axillary lymph node also indicated metastasis. The patient underwent a mastectomy at a tertiary hospital with a postoperative stage of T3N2 (primary tumor: $7 \mathrm{~cm} \times 5 \mathrm{~cm}$ $\times 3.5 \mathrm{~cm}$; axillary lymph nodes: 4/10), followed by 6 cycles of adjuvant chemotherapy (pirarubicin $60 \mathrm{mg}$, cisplatin $100 \mathrm{mg}$, and 5-Fu $2.5 \mathrm{~g}$ ). The patient refused radiotherapy and endocrine therapy.

In March 2015, the patient suffered from disease progression with pulmonary, pleural, and sternal metastasis, but declined target therapy and chemotherapy. The treatment strategy was switched to a bilateral oophorectomy followed by letrozole and ZA. In February 2017, the patient experienced a second progression of pulmonary metastases.
She was then enrolled in the HLX02-BC01 clinical trial and underwent 8 cycles of HLX02 (trastuzumab made in China, $8 \mathrm{mg} / \mathrm{kg}$ first dose and $6 \mathrm{mg} / \mathrm{kg}$ in maintenance) plus docetaxel $\left(75 \mathrm{mg} / \mathrm{m}^{2}\right)$ every 3 weeks.

In November 2017, the patient showed further disease progression of pulmonary metastasis. At this time, her HER-2 status was rechecked and was found to be positive. She was then enrolled into the BO29919 clinical trial. The patient underwent 12 cycles of trastuzumab emtansine (TDM-1). Her response was evaluated as partial remission (PR), but TDM-1 was suspended due to grade 3 thrombocytopenia.

In October 2018, the patient complained of progressive sensory disturbance and a mobility disorder in a right limb. Subsequent brain magnetic resonance imaging (MRI) revealed metastases. Local radiotherapy to the metastatic brain lesions was initiated from October 18, 2018, at a dose of 45 Gy/5 Gy/9 f. The patient also took $250 \mathrm{mg}$ of apatinib, a small-molecular TKI that targets intracellular segments of vascular endothelial growth factor receptor 2 (VEGFR2), daily from October 15, 2018. However, surprisingly, in October 26, 2018, massive bleeding from the oral and nasal cavity occurred. A computed tomography (CT) scan revealed severe destruction and loss of the right maxillary bone and maxillary sinus medial and lateral wall (see Figure $1 A-1 C$ ). A pathological examination of the exfoliated bone tissue demonstrated bone necrosis, and BM was excluded (see Figure 1D). The bleeding continued despite aggressive medical hemostasis and tamponade treatment. Embolization hemostasis treatment was then recommended. Intraoperative arteriography revealed the presence of contrast agent dispersion from the upper alveolar branch of the right internal mandibular artery. An emergency interventional embolization was performed, and the bleeding was stopped. Anti-infective treatment was given after the operation. The patient received ZA regularly (once a month) from March 2015 to April 2018 and denied any history of tooth extraction, oral operation, or local debridement. However, in 2016, she suffered from a toothache and underwent analgesic treatment and took an antibiotic (ornidazole). Thus, the ZA and apatinib treatment was ceased. Fulvestrant was administered from October 30, 2018, to April 26, 2019. This patient was heavily treated and her prognosis was poor.

\section{Follow-up and outcomes}

In May 2019, the patient's intracranial metastasis had 


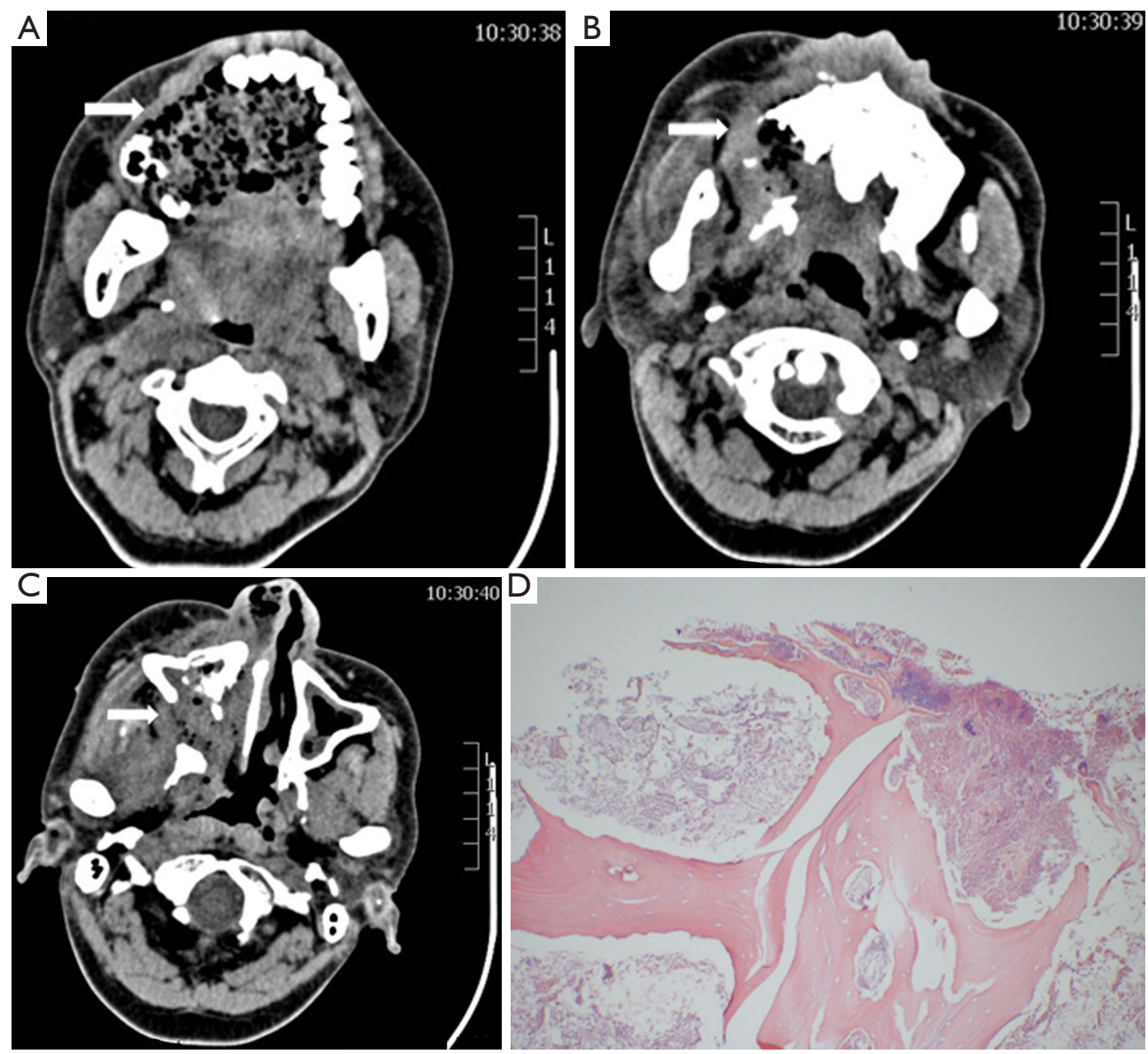

Figure 1 Maxillofacial computed tomography (CT) and pathological findings. Maxillofacial CT demonstrated severe destruction and loss of the right maxillary bone and maxillary sinus medial and lateral wall. (A) Right maxillary bone image. (B) Right maxillary bone image. (C) Right maxillary sinus medial and lateral wall image. White arrow (A-C) indicated bone defect. (D) The pathology of the exfoliated bone tissue showed no osteocytes in the lacunae, indicating bone necrosis (HE staining after decalcification, original magnification, 100×).

progressed. A brain MRI showed metastasis of the left temporal parietal lobe. The lesion of the left parietal lobe appeared to be enlarged. A new treatment strategy of chemotherapy with pirlotinib and capecitabine was employed. In August 2019, the patient refused any antitumor management. The local necrosis and defects became progressively aggravated, causing the mouth and nasal cavity to communicate. She found it difficult to eat and drink and often choked after drinking. The patient died on July 24, 2020 (see Figure 2).

All procedures performed in studies involving human participants were in accordance with the ethical standards of the institutional and/or national research committee(s) and with the Helsinki Declaration (as revised in 2013). Written informed consent was obtained from the patient's son for publication of this case report and accompanying images. A copy of the written consent is available for review by the editorial office of this journal.

\section{Discussion}

Bone is one of the most common metastatic sites for solid tumors (3). Bone metastases develop in approximately 54 $81 \%$ of prostate cancer patients $(4,5), 55 \%$ of breast cancer patients (6), $20-40 \%$ of lung cancer patients, and $17.4 \%$ of renal cell carcinoma patients $(7,8)$. Skeletal-related events caused by BM include pathologic fracture, spinal cord compression, hypercalcemia, and local pain (3). These events not only seriously reduce patients' quality of life but also negatively affect their prognosis (3). Antiresorptive 


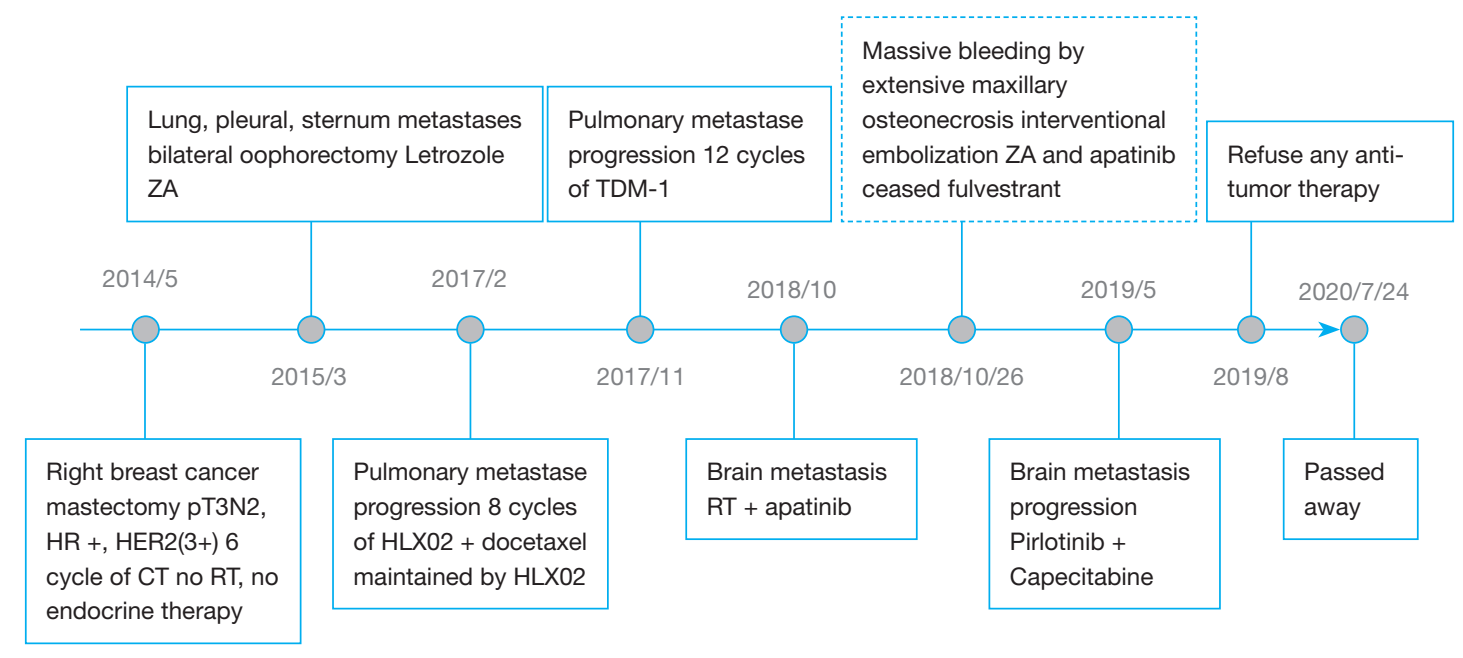

Figure 2 The timeline of diagnosis, treatment, and follow-up.

agents, such as ZA, are the standard option for treating BM.

With an occurrence rate of $1 \%$, ZA-related osteonecrosis of the jaw $(\mathrm{ONJ})$ is rare (9). In cancer patients, the incidence of $\mathrm{ONJ}$ related to $\mathrm{BP}$ therapy has been reported to be between $0.8-3.1 \%$ (10-14); however, its incidence increases when it is used combination with antiangiogenic drugs (2) (see Table 1). The incidence of ONJ differs in various tumors. In a retrospective study, the incidence of BPrelated $\mathrm{ONJ}$ in patients with multiple myeloma and breast cancer was higher than that in patients with prostate cancer, lung cancer, kidney cancer, and other tumors (12). To be diagnosed with $\mathrm{ONJ}$, a patient must meet the following criteria proposed by the American Association of Oral and Maxillofacial Surgeons (AAOMS): (I) a persistently nonhealing mandible or maxillary bone for longer than 8 weeks, (II) treatment with antiresorptive agents and/or antiangiogenesis, (III) no history of previous radiation to this region, and (IV) no tumor invasion or metastasis in this site $(13,15,16)$.

Our patient was differentiated from BM, suppurative osteomyelitis, and radiation osteomyelitis. The patient had no obvious symptoms, such as a high fever or chills, and the results of the laboratory examination excluded suppurative osteomyelitis. Radiation osteomyelitis was also excluded, as the patient had no history of radiotherapy in the maxillofacial region. MRONJ patients have a clear history of related drug use. In our case, a CT scan revealed obvious destruction and loss of the right maxillary bone and maxillary sinus walls. The patient had taken ZA for a long period ( 37 months) and had also taken apatinib.
The pathology of the exfoliated bone tissue confirmed bone necrosis. Thus, the patient was diagnosed with osteonecrosis.

Qi et al. retrospectively reviewed 2,214 individuals with advanced BM cancer from 3 phase III clinical trials. The multivariate analysis revealed that old age $(\geq 66 ; \mathrm{P}=0.007)$, anemia $(\mathrm{P}=0.006)$, and a long duration of $\mathrm{ZA}$ application $(1-2$ years; $\mathrm{P}=0.01 ; \geq 2$ years; $\mathrm{P}<0.001)$ were closely associated with a high risk of osteonecrosis (13). Our patient had used ZA for a rather long period (37 months), which contributed greatly to the severe osteonecrosis and results in massive bleeding. In the OPTIMIZE-2 clinical trial, 2 patients developed ONJ in the every-4-week group, while no patient developed ONJ in the every-12-week group (17). The question of whether tooth extraction leads to a high risk of developing ONJ remains controversial. Hasegawa et al. suggested that tooth extraction is a major risk factor of ONJ (18). Similarly, Kyrgidis et al. found that tooth extraction led to a 16-fold increase in the risk of ZA-related ONJ (19). Despite having no history tooth extraction, a history of oral infection in our patient partly aggravated the development of osteonecrosis. Lipopolysaccharide, a bacterial cell wall constituent, has been shown to enhance the direct necrotic effects of ZA (20). Over 800 kinds of bacteria inhabit the human oral mucosa (21). If oral mucosal damage occurs, these bacteria can easily reach the jaw bones and contribute to bone necrosis.

Antiresorptive and antiangiogenetic agents may share a common pathological process in inducing osteonecrosis. The key mechanism includes blocking the VEGF/VEGFR 


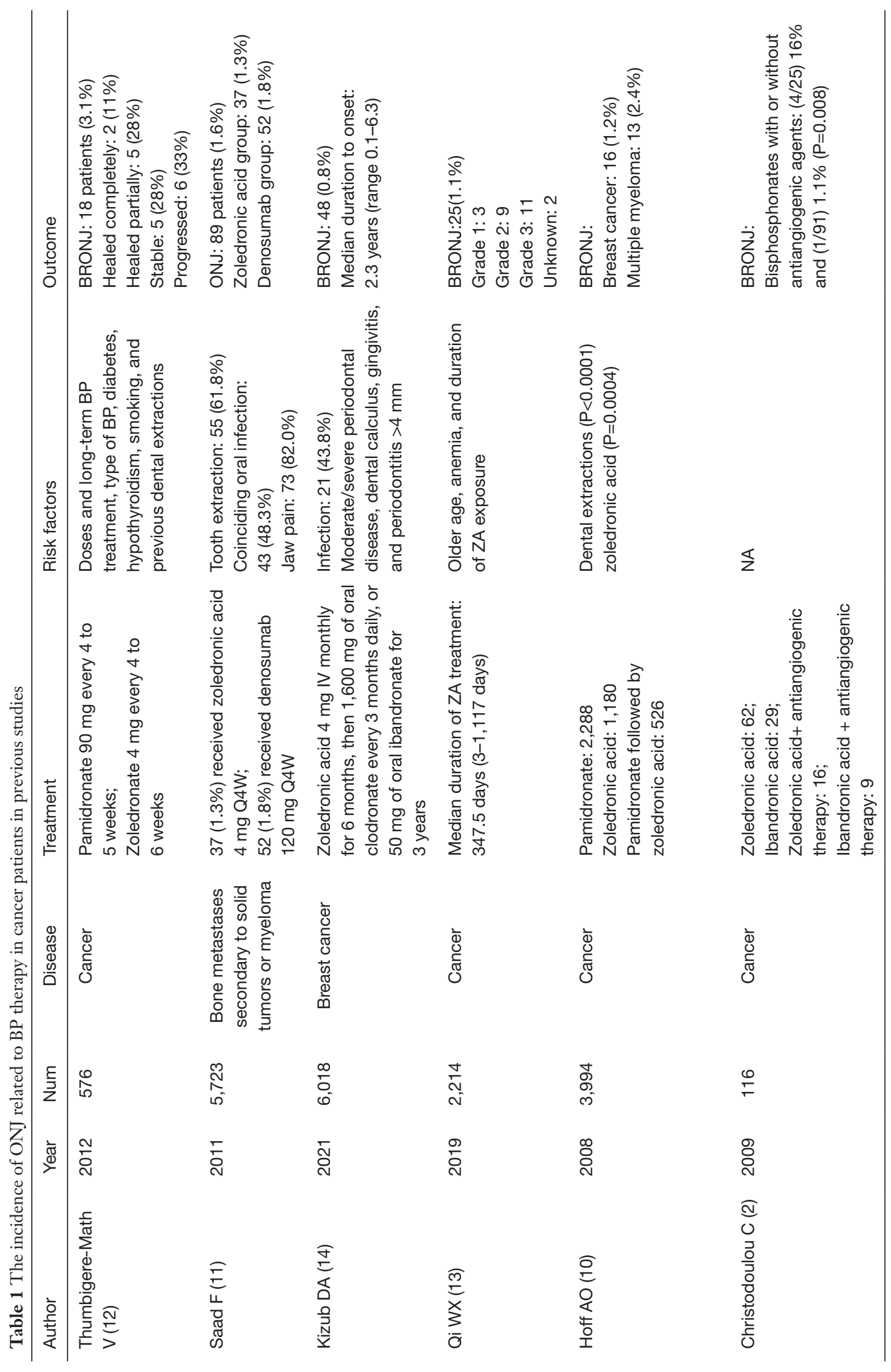


pathway, which results in the inhibition of proliferation, migration, permeability, and the survival of vascular endothelial cells (VEC). Allegra et al. found that application of $\mathrm{BP}$ resulted in a decrease in circulating endothelial progenitor cells (EPCs) and thus interfered with endothelial cell proliferation $(20,22)$. Additionally, BP administration has been shown to induce endothelial cell apoptosis $(20,23)$. Nitrogen-containing bisphosphonates (NBPs) can also inhibit the expression of osteopontin in the mandible, which in turn promotes the proliferation of endothelial cells (24).

NBPs inhibit the number and function of $\gamma \delta \mathrm{T}$ lymphocytes in circulation and reduce the serum levels of interleukin 17, which plays a critical role in the stimulation of VEC migration and the secretion of proangiogenic factors $(20,24,25)$. Further, NBPs directly inhibit the messenger RNA (mRNA) expression of VEGF and thus reduce the production of VEGF (26). NBPs inhibit the activity of osteoclasts, preventing osteoclast from releasing stored VEGF and indirectly inhibiting VEGF production (27). NBPs inhibit the activity of matrix metalloproteinase 9 in the extracellular matrix, thereby reducing VEGF-VEGFR binding and inhibiting angiogenesis (28).

In 2008, Estilo et al. were first to report a patient with breast cancer and a patient with glioblastoma multiforme developing $\mathrm{ONJ}$ after receiving the antiangiogenic drug, bevacizumab (29). A subsequent retrospective study found that patients receiving $\mathrm{BP}$ combined with antiangiogenic drugs had a higher incidence of ONJ than did patients receiving $\mathrm{BP}$ alone $(16 \%$ vs. $1.1 \% ; \mathrm{P}=0.008)$ (2). Lescaille et al. reviewed $42 \mathrm{ONJ}$ patients and found that $\mathrm{ZA}$ combined with bevacizumab was associated with an increased risk of spontaneous ONJ (odds ratio: 6.07; 95\% confidence interval: $1.3-28.2 ; \mathrm{P}<0.05)(30)$. Ngamphaiboon et al. found that patients receiving BPs combined with antiangiogenic drugs had more severe symptoms, such as pain, abscesses, and loose teeth than patients with BPs alone (31). Apatinib, a potent small-molecule inhibitor of VEGFR2 signaling, undoubtedly exerts a synergistic action on BP in avascular necrosis. Additionally, local infection and trauma can also aggravate $\mathrm{ONJ}$.

The treatment of ONJ is rather challenging, and no standard paradigm has thus far been established. According to the AAOMS, ONJ has 4 stages (9). Conservative treatments, such as antibiotics and analgesics, are generally applied to patients in the early stage (stage $0-1$ ), while surgery has been shown to be superior to supportive management alone in more advanced patients (in stages
2-3) (9). However, the complete removal of the necrotic bones and closure of the wound are critical to the success of surgery $(32,33)$. In our case, the involved region was so extensive that the complications caused by the surgery were severe. Providing new insights into their role in ONJ, Steller et al. reported that platelet-rich plasma and plateletrich fibrin full of growth factors, such as platelet-derived growth factor, transforming growth factor, and insulinlike growth factor, reduce the negative effects of $\mathrm{ZA}$ on cell viability, proliferation, and migration (34).

The findings of our case are noteworthy in many respects. First, the combined use of NBP and angiogenetic drugs is rather common in clinical practice, but the subsequent disastrous complications, such as osteonecrosis, which occurred in our patient, have failed to attract sufficient attention. Populations at high-risk of osteonecrosis should be identified, and the intensity and duration of treatments should be adjusted correspondingly to ensure that severe side effects are avoided where possible. An oral inspection should be performed before BP or antiangiogenesis drugs are administered and unnecessary oral procedures should be avoided during treatment. Second, we analyzed the mechanism of osteonecrosis induced by NBP and angiogenetic agents. In the future, novel drugs targeting the key link in osteonecrosis development should be addressed to solve the current therapeutic dilemmas facing patients with osteonecrosis. Third, care needs to be taken to ensure that osteonecrosis is not mistakenly diagnosed as BM in heavily-treated cancer patients with a definite history of metastases in the bone and/or other sites. Pathology is essential to ensuring the correct diagnosis and management.

In conclusion, given the common application of antiresorptive and antiangiogenetic agents, severe side effects, such as the extensive osteonecrosis shown in our case, should be avoided. The high-risk patients, causes, underlying molecular mechanism, and best intervention for drug-related osteonecrosis need to be further clarified. Pathology is a prerequisite for excluding BM, especially in refractory and metastatic settings. This issue should be given more attention, as it seriously affects patients' quality of life.

\section{Acknowledgments}

The authors acknowledge AME Editing Service for editing the manuscript.

Funding: None. 


\section{Footnote}

Reporting Checklist: The authors have completed the CARE reporting checklist. Available at https://dx.doi. org/10.21037/tcr-21-404

Peer Review File: Available at https://dx.doi.org/10.21037/ tcr-21-404

Conflicts of Interest: All authors have completed the ICMJE uniform disclosure form (available at https://dx.doi. org/10.21037/tcr-21-404). The authors have no conflicts of interest to declare.

Ethical Statement: The authors are accountable for all aspects of the work, including ensuring that all questions related to the accuracy or integrity of any part of the work have been appropriately investigated and resolved. All procedures performed in studies involving human participants were in accordance with the ethical standards of the institutional and/or national research committee(s) and with the Helsinki Declaration (as revised in 2013). Written informed consent was obtained from the patient's son for publication of this case report and accompanying images. A copy of the written consent is available for review by the editorial office of this journal.

Open Access Statement: This is an Open Access article distributed in accordance with the Creative Commons Attribution-NonCommercial-NoDerivs 4.0 International License (CC BY-NC-ND 4.0), which permits the noncommercial replication and distribution of the article with the strict proviso that no changes or edits are made and the original work is properly cited (including links to both the formal publication through the relevant DOI and the license). See: https://creativecommons.org/licenses/by-nc-nd/4.0/.

\section{References}

1. Ouyang Z, Li H, Zhai Z, et al. Zoledronic Acid: Pleiotropic Anti-Tumor Mechanism and Therapeutic Outlook for Osteosarcoma. Curr Drug Targets 2018;19:409-21.

2. Christodoulou C, Pervena A, Klouvas G, et al. Combination of bisphosphonates and antiangiogenic factors induces osteonecrosis of the jaw more frequently than bisphosphonates alone. Oncology 2009;76:209-11.

3. Turpin A, Duterque-Coquillaud M, Vieillard MH.
Bone Metastasis: Current State of Play. Transl Oncol 2020;13:308-20.

4. Gravis G, Fizazi K, Joly F, et al. Androgen-deprivation therapy alone or with docetaxel in non-castrate metastatic prostate cancer (GETUG-AFU 15): a randomised, openlabel, phase 3 trial. Lancet Oncol 2013;14:149-58.

5. James ND, Sydes MR, Clarke NW, et al. Addition of docetaxel, zoledronic acid, or both to first-line longterm hormone therapy in prostate cancer (STAMPEDE): survival results from an adaptive, multiarm, multistage, platform randomised controlled trial. Lancet 2016;387:1163-77.

6. Body JJ, Quinn G, Talbot S, et al. Systematic review and meta-analysis on the proportion of patients with breast cancer who develop bone metastases. Crit Rev Oncol Hematol 2017;115:67-80.

7. Kuchuk M, Addison CL, Clemons M, et al. Incidence and consequences of bone metastases in lung cancer patients. $\mathrm{J}$ Bone Oncol 2013;2:22-9.

8. Motzer RJ, Hutson TE, Cella D, et al. Pazopanib versus sunitinib in metastatic renal-cell carcinoma. N Engl J Med 2013;369:722-31.

9. Ruggiero SL, Dodson TB, Fantasia J, et al. American Association of Oral and Maxillofacial Surgeons position paper on medication-related osteonecrosis of the jaw-2014 update. J Oral Maxillofac Surg 2014;72:1938-56.

10. Hoff AO, Toth BB, Altundag K, et al. Frequency and risk factors associated with osteonecrosis of the jaw in cancer patients treated with intravenous bisphosphonates. J Bone Miner Res 2008;23:826-36.

11. Saad F, Brown JE, Van Poznak C, et al. Incidence, risk factors, and outcomes of osteonecrosis of the jaw: integrated analysis from three blinded active-controlled phase III trials in cancer patients with bone metastases. Ann Oncol 2012;23:1341-7.

12. Thumbigere-Math V, Tu L, Huckabay S, et al. A retrospective study evaluating frequency and risk factors of osteonecrosis of the jaw in 576 cancer patients receiving intravenous bisphosphonates. Am J Clin Oncol 2012;35:386-92.

13. Qi WX, Zhao S, Chen J. Risk factors for developing osteonecrosis of jaw in advanced cancer patients underwent zoledronic acid treatment. Future Oncol 2019;15:3503-11.

14. Kizub DA, Miao J, Schubert MM, et al. Risk factors for bisphosphonate-associated osteonecrosis of the jaw in the prospective randomized trial of adjuvant bisphosphonates for early-stage breast cancer (SWOG 0307). Support Care Cancer 2021;29:2509-17. 
15. Marx RE, Sawatari Y, Fortin M, et al. Bisphosphonateinduced exposed bone (osteonecrosis/osteopetrosis) of the jaws: risk factors, recognition, prevention, and treatment. J Oral Maxillofac Surg 2005;63:1567-75.

16. Altay MA DDS, PhD, Radu A DMD, Pack SE DDS, MD, et al. Medication-related osteonecrosis of the jaw: An institution's experience. Cranio 2020;38:333-41.

17. Hortobagyi GN, Van Poznak C, Harker WG, et al. Continued Treatment Effect of Zoledronic Acid Dosing Every 12 vs 4 Weeks in Women With Breast Cancer Metastatic to Bone: The OPTIMIZE-2 Randomized Clinical Trial. JAMA Oncol 2017;3:906-12.

18. Hasegawa T, Hayashida S, Kondo E, et al. Medicationrelated osteonecrosis of the jaw after tooth extraction in cancer patients: a multicenter retrospective study. Osteoporos Int 2019;30:231-9.

19. Kyrgidis A, Vahtsevanos K, Koloutsos G, et al. Bisphosphonate-related osteonecrosis of the jaws: a casecontrol study of risk factors in breast cancer patients. J Clin Oncol 2008;26:4634-8.

20. Allegra A, Innao V, Pulvirenti N, et al. Antiresorptive Agents and Anti-Angiogenesis Drugs in the Development of Osteonecrosis of the Jaw. Tohoku J Exp Med 2019;248:27-9.

21. Allegra A, Innao V, Allegra AG, et al. Role of the microbiota in hematologic malignancies. Neth $\mathrm{J}$ Med 2019;77:67-80.

22. Allegra A, Oteri G, Nastro E, et al. Patients with bisphosphonates-associated osteonecrosis of the jaw have reduced circulating endothelial cells. Hematol Oncol 2007;25:164-9.

23. Allegra A, Alonci A, Penna G, et al. Bisphosphonates induce apoptosis of circulating endothelial cells in multiple myeloma patients and in subjects with bisphosphonateinduced osteonecrosis of the jaws. Acta Haematol 2010;124:79-85.

24. Chang J, Hakam AE, McCauley LK. Current Understanding of the Pathophysiology of Osteonecrosis of the Jaw. Curr Osteoporos Rep 2018;16:584-95.

25. Oteri G, Allegra A, Bellomo G, et al. Reduced serum levels of Interleukin 17 in patients with osteonecrosis of the jaw and in multiple myeloma subjects after bisphosphonates administration. Cytokine 2008;43:103-4.

26. Santini D, Vincenzi B, Galluzzo S, et al. Repeated intermittent low-dose therapy with zoledronic acid induces an early, sustained, and long-lasting decrease of peripheral vascular endothelial growth factor levels in cancer patients. Clin Cancer Res 2007;13:4482-6.

27. Forte L, Torricelli P, Boanini E, et al. Antiresorptive and anti-angiogenetic octacalcium phosphate functionalized with bisphosphonates: An in vitro tri-culture study. Acta Biomater 2017;54:419-28.

28. Giraudo E, Inoue M, Hanahan D. An aminobisphosphonate targets MMP-9-expressing macrophages and angiogenesis to impair cervical carcinogenesis. J Clin Invest 2004;114:623-33.

29. Estilo CL, Fornier M, Farooki A, et al. Osteonecrosis of the jaw related to bevacizumab. J Clin Oncol 2008;26:4037-8.

30. Lescaille G, Coudert AE, Baaroun V, et al. Clinical study evaluating the effect of bevacizumab on the severity of zoledronic acid-related osteonecrosis of the jaw in cancer patients. Bone 2014;58:103-7.

31. Ngamphaiboon N, Frustino JL, Kossoff EB, et al. Osteonecrosis of the jaw: dental outcomes in metastatic breast cancer patients treated with bisphosphonates with/ without bevacizumab. Clin Breast Cancer 2011;11:252-7.

32. Kagami H, Inoue M, Kobayashi A, et al. Issues with the surgical treatment of antiresorptive agent-related osteonecrosis of the jaws. Oral Dis 2018;24:52-6.

33. Khan AA, Morrison A, Hanley DA, et al. Diagnosis and management of osteonecrosis of the jaw: a systematic review and international consensus. J Bone Miner Res 2015;30:3-23.

34. Steller D, Herbst N, Pries R, et al. Positive impact of Platelet-rich plasma and Platelet-rich fibrin on viability, migration and proliferation of osteoblasts and fibroblasts treated with zoledronic acid. Sci Rep 2019;9:8310.

(English Language Editors: L. Huleatt and J. Gray)
Cite this article as: Liu B, Ma Y, Liu H, Wang C, Guo L, Shi A, Liu M. Unexpected massive bleeding caused by extensive maxillary osteonecrosis in a breast cancer patient: a case report. Transl Cancer Res 2021;10(11):5014-5021. doi: 10.21037/tcr21-404 